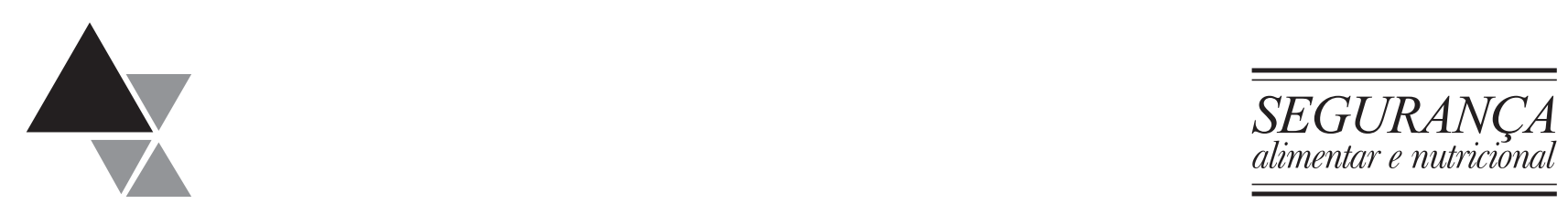

\title{
CONSUMO DE FRUTOSE E IMPACTO NA SAÚDE HUMANA
}

\author{
Natalia Moreno Gaino ${ }^{1}$ Marina Vieira da Silva ${ }^{2}$
}

Determinados açúcares de adição dos alimentos industrializados são alvos de estudos que relacionam o seu consumo excessivo com o aumento da obesidade e comorbidades associadas como o diabetes, câncer, dislipidemia e aterosclerose. A identificação da quantidade dessa modalidade de açúcares na composição dos alimentos e impacto na saúde humana decorrentes do consumo excessivo constituem informações fundamentais para a promoção da saúde e da segurança alimentar e nutricional. A frutose é comumente encontrada como um componente natural em determinados alimentos ou como um ingrediente adicional (como a sacarose e xarope de milho rico em frutose). O consumo de frutose aumentou drasticamente nas últimas 4 décadas, devido ao seu uso generalizado em muitos alimentos e bebidas (frutas enlatadas, geleias, doces em pasta, bolos, pudins, tabletes, pó para bebidas, refrigerantes). Pesquisas sobre o excesso de peso vêm sugerindo que o alto consumo de frutose poderia estar relacionado ao desenvolvimento da síndrome metabólica. Acervos de estudos consideram que a ingestão elevada de frutose pode induzir a alterações do metabolismo, como o aumento de triglicérides, resistência insulínica e esteatose hepática. Porém, esses resultados não são conclusivos. Pesquisas envolvendo intervenções de longo prazo e nas quais sejam empregadas quantidades de açúcares normalmente ingeridas pelos indivíduos são necessárias e podem preencher a lacuna de informações sobre o tema. Políticas públicas poderiam contribuir para a definição e monitoramento visando limitar o consumo dos alimentos ricos em açúcares de adição. Para a população devem ser fornecidos subsídios para que realizem escolhas alimentares mais saudáveis, o que contribuiria para o alcance da segurança alimentar e nutricional.

Palavras-chave: frutose, alimentos industrializados, doenças crônicas, segurança alimentar e nutricional.

\section{FRUCTOSE CONSUMPTION AND IMPACT ON HUMAN HEALTH}

Some added sugars in processed foods are targets of studies linking excessive consumption with increased obesity and associated comorbidities such as diabetes, cancer, dyslipidemia and atherosclerosis. The identification of the quantity of this type of sugars in the food composition and impact on human health arising from excessive consumption are key information for health promotion and food security and nutrition. Fructose is commonly found as a natural component of certain foods or as an additional ingredient (such as sucrose and corn syrup rich in fructose). Fructose consumption has increased dramatically in the last four decades due to its widespread use in many foods and beverages (canned fruits, jams, sweet pastries, cakes, puddings, tablets, powdered drinks, soda). Research on excess weight have suggested that high fructose consumption could be related to the development of metabolic syndrome. Collections of studies find that high intake of fructose can induce metabolic changes such as increased triglycerides, insulin resistance and hepatic steatosis. However, these results are not conclusive. Research involving long-term interventions and which are employed in amounts of sugars ingested by individuals are necessary and can fill the information gap on the subject. Public policies could contribute to defining and

\footnotetext{
${ }^{1}$ Mestranda do Programa de Pós-graduação em Ciência e Tecnologia de Alimentos - Escola Superior de Agricultura Luiz de Queiroz (ESALQ), Universidade de São Paulo (USP), Depto. de Agroindústria, Alimentos e Nutrição, Piracicaba, SP. Bolsista FAPESP (Número do processo: 2010/06800-0). Correspondência: Rua Guaporé 340, Apt. 48, Piracicaba, SP, CEP:13417-290 E-mail: natigaino@yahoo.com.br.

${ }^{2}$ Profa. Dra., Depto. de Agroindústria, Alimentos e Nutrição, ESALQ (USP), Piracicaba, SP .
} 
monitoring to limit the consumption of foods high in added sugars. For the population should be provided subsidies for carrying out healthier food choices, which contribute to the achievement of food security and nutrition.

Key-words: fructose, processed foods, chronic diseases, food safety and nutrition.

\section{INTRODUÇÃO}

Em decorrência do processo de transição nutricional, em curso no Brasil, a obesidade surge como uma importante variável que contribui para o comprometimento do estado nutricional da população. As consequências em longo prazo da obesidade associam-se a problemas debilitantes da saúde, além de constituir fator de risco para Doenças Crônicas Não Transmissíveis (DCNT), entre elas, o diabetes mellitus, hipertensão arterial, dislipidemias, doenças cardiovasculares e câncer ${ }^{[1]}$.

As mudanças nos padrões dietéticos que caracterizam a chamada transição nutricional envolvem alterações quantitativas e qualitativas na dieta. As novas práticas alimentares incluem elevado conteúdo energético, com maior participação de gorduras e açúcares de adição, crescimento no consumo de gordura saturada (maior parte de origem animal) e redução no consumo de fibra dietética, frutas, hortaliças e cereais ${ }^{[2]}$.

Em 1996, o Departamento de Agricultura americano definiu o termo açúcares de adição que inclui além dos monos e dissacarídeos, alguns oligossacarídeos e não considera os açúcares naturalmente presentes em alimentos, como é o caso dos leites e frutas. Esses açúcares são aqueles acrescentados em preparações e alimentos processados com o objetivo de provocar paladar agradável, melhor viscosidade, textura, cor e durabilidade. Incluem-se neste termo açúcar refinado, açúcar mascavo, xarope de milho rico em frutose, xarope de glicose, frutose líquida, edulcorante a base de frutose, mel e melaço ${ }^{[3]}$.

De acordo com a Pesquisa de Orçamentos Familiares (POF) 2008-2009, a disponibilidade relativa dos macronutrientes evidencia excesso do teor de açúcar para as famílias de cinco regiões geográficas (variando de 13,9\% das calorias totais na Região Norte a $17,4 \%$ na Região Sudeste), sendo o limite máximo de $10 \%$ para a proporção de calorias provenientes de açúcares largamente ultrapassado em todas as Regiões e classes de rendimentos, além de um aumento de $16 \%$ para os refrigerantes em sua participação no total de calorias ${ }^{[4]}$. Levy-Costa et al. ${ }^{[5]}$ na análise da evolução da disponibilidade domiciliar de alimentos no Brasil, tendo como base os dados da POF 20022003, encontraram aumento no consumo per capita de refrigerante $(+400 \%)$ para famílias brasileiras pertencentes a todas as categorias de rendimento. Entre os produtos que apresentaram aumento de suas quantidades per capita médias adquiridas entre os períodos de realização das POFs nacionais (2002/2003 - 2008/2009) destaca-se o refrigerante de cola $(39,3 \%)$. Considerando-se a situação do domicílio, na área rural, esse aumento foi ainda mais expressivo. A quantidade média adquirida do refrigerante de cola passou de 3,2 kg para 6,1 kg, representando aumento de $92 \%$ no período considerado ${ }^{[4]}$.

O consumo excessivo de refrigerante pode provocar aumento de peso corporal. A possível explicação é que a energia obtida por líquidos provoca menor saciedade que a obtida por alimentos sólidos. O consumo de refrigerantes, constituído basicamente de açúcar de adição ou edulcorantes na versão light/ diet, está associado também à redução da ingestão de leite e sucos de frutas naturais ${ }^{[6]}$.

Nos Estados Unidos, há uma preocupação com relação à presença em excesso de produtos a base de açúcares refinados na dieta. Também foram observados aumentos alarmantes no consumo desses produtos em diversos países em desenvolvimento como China, Índia, Vietnã, Tailândia e outros países do sudeste asiático, os quais representam atualmente um forte mercado para a indústria de refinados ${ }^{[7]}$.

Do ponto de vista nutricional, Lewis et al. ${ }^{[8]}$ observaram que pessoas que ingeriam maior percentual de energia provenientes dos açúcares de adição consumiam 11 vitaminas e minerais em menor quantidade. Boowman ${ }^{[9]}$ mostrou que indivíduos com participação na dieta de $18 \%$ ou mais da energia proveniente de açúcares de adição apresentavam maior consumo de energia e menor ingestão de proteínas, fibras e da maioria dos micronutrientes. Esse comportamento alimentar contribui decisivamente para ampliar os riscos de desenvolvimento de DCNT. 
Os açúcares de adição podem ser um veículo para uma dieta rica em gordura devido a uma relação positiva entre estas substâncias. Alimentos como bolos, biscoitos, chocolates e outras sobremesas são fontes de açúcares assim como gorduras e, consequentemente, energia. A maior parte desta gordura é saturada, contendo ácidos graxos trans devido à utilização da gordura hidrogenada nestas preparações. Portanto, o consumo exagerado destes alimentos é um fator de risco para a obesidade e suas complicações como diabetes e doenças cardiovasculares ${ }^{[10]}$.

Determinados açúcares de adição dos alimentos industrializados, são alvos de estudos que relacionam o seu consumo excessivo com o aumento da obesidade e comorbidades associadas como o diabetes, câncer, dislipidemia e aterosclerose. Nesta perspectiva, seu consumo excessivo merece destaque, por estar negativamente associado à ingestão de nutrientes essenciais e à qualidade da dieta, podendo contribuir para o substancial consumo energético e, por consequência, para o aumento de peso ${ }^{[11]}$.

Com a introdução na dieta, no final da década de 1960, do xarope de milho rico em frutose, principal açúcar utilizado para adoçar alimentos industrializados nos Estados Unidos, a presença de frutose na dieta americana aumentou notavelmente, contribuindo para o desenvolvimento de resistência à insulina, obesidade, hipertensão e anormalidades lipídicas. A partir desses dados, a busca por modelos animais com sobrecarga de frutose e que levassem a alterações semelhantes passou a motivar os pesquisadores da área. De fato, ratos alimentados com frutose mostraram moderada hipertensão e intolerância à glicose, associados a níveis plasmáticos aumentados de insulina, colesterol e triglicérides. Em camundongos, o consumo crônico de frutose alterou o metabolismo de glicose e o perfil lipídico ${ }^{[12]}$.

A identificação da quantidade de açúcares de adição na composição dos alimentos e os impactos na saúde humana decorrentes do consumo em excesso, constituem informações fundamentais para elaboração e adoção de estratégias que contribuam para a promoção da saúde e também da segurança alimentar e nutricional da população.

Face ao exposto, o presente artigo visou, por meio de revisão da literatura pertinente, conhecer o consumo de açúcares de adição, principalmente a frutose, pela população, bem como avaliar os impactos que o consumo excessivo desses açúcares pode causar na saúde humana. Estas informações podem auxiliar na formulação de políticas públicas que visem ampliar o acesso à segurança alimentar e nutricional.

\section{FRUTOSE}

A frutose, também chamada de levulose (uma solução saturada capaz de transformar luz linearmente polarizada em luz circularmente polarizada, com giro vetorial para esquerda), é constituinte da sacarose ( $\beta$-D-frutofuranosil $\alpha$-D-glicopiranosida) e de outros polímeros denominados fructans ou inulina, além de ser encontrado sob a forma isolada na natureza [13,14]. Está presente nas frutas, mel (constituído de frutose e glicose em iguais proporções), xarope de frutose (produzido a partir da hidrólise da sacarose proveniente do melaço da cana-de-açúcar), xarope de milho (com alta concentração de frutose), dentre outros ${ }^{[13]}$. É o monossacarídeo predominante em várias frutas, incluindo maçãs, laranjas e melões. Pode ser encontrada como rafinose, que é um trissacarídeo, e também como estaquiose, que é um tetrassacarídeo, em algumas leguminosas como a soja, lentilha, ervilha e feijão ${ }^{[14]}$.

A partir do sorbitol a frutose pode ser produzida e a reação ocorre quando esse poliól é oxidado em uma reação mediada pela enzima sorbitol-desidrogenase. O sorbitol está presente em várias plantas, em particular na família das Rosáceas (maçãs, peras, cerejas, ameixas e abricós), geralmente associado à frutose. Outras fontes de sorbitol são doces, balas de menta, goma de mascar, alimentos e sucos dietéticos ${ }^{[14]}$.

A frutose tem sido incorporada com sucesso nas fórmulas envolvidas no preparo de frutas enlatadas, geleias, doces em pasta, bolos, pudins, tabletes, pó para bebidas, refrigerantes etc., uma vez que é 1,5 vezes mais doce do que a sacarose. Seu custo vem sendo reduzido gradualmente ao longo dos anos como resultado da melhoria nos processos envolvidos na obtenção. Sua utilização em produtos que não tenham aplicação alimentar é reduzida, o que não chega a ser uma surpresa já que a química básica relacionada a este carboidrato é muito pouco desenvolvida quando comparada à glicose ${ }^{[15]}$. 
Baseando-se nas fontes de açúcares da dieta da parcela da população que envolve americanos a partir dos 2 anos de idade (Figura 1) é possível reconhecer que, os alimentos que fornecem maiores quantidades de açúcares de adição são frequentemente alimentos com baixa densidade de outros nutrientes, como refrigerantes e sobremesas.

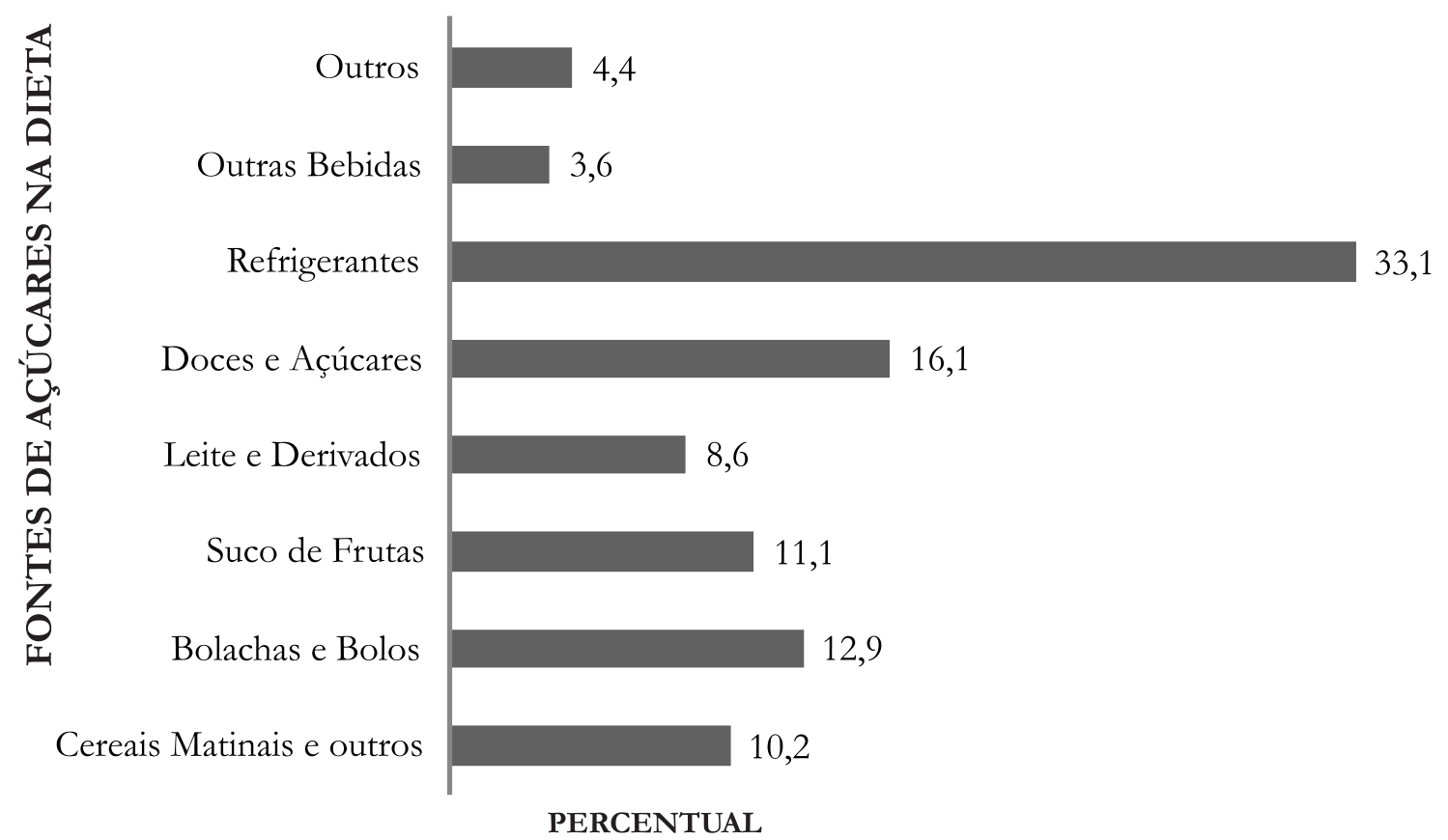

Figura 1. Fontes de açúcares de adição na dieta de americanos a partir dos dois anos de idade. Adaptado de Guthrie \& Morton ${ }^{[16]}$.

O comitê formado para discutir o guia alimentar americano apontou para a necessidade da distinção da recomendação dos açúcares de adição daquela naturalmente encontrada em alimentos. No entanto, a conclusão foi que não havia justificativa científica suficiente para adotar o termo açúcares de adição no guia e rótulos de alimentos. A dificuldade que empresas alimentícias teriam para diferenciar nos produtos a quantidade de açúcares de adição e o naturalmente presente também influenciou na decisão de manter a informação de açúcares totais nos rótulos, considerando que são açúcares quimicamente e fisiologicamente semelhantes ${ }^{[3,17]}$.

\section{CONSUMO DE FRUTOSE}

Dados coletados a partir do estudo NHANES 1999-2004 estimaram uma ingestão de frutose média de $49 \mathrm{~g} /$ dia para a população americana. Foi também documentado que o consumo de xarope de milho rico em frutose aumentou continuamente ao longo das últimas três décadas e foi responsável por $42 \%$ do total do consumo de edulcorantes calóricos no período definido pelos anos de 1999 e 2004 versus 16\% entre os anos de 1977 e 1978. Curiosamente, esta análise também mostrou que o consumo total de energia e a ingestão de carboidrato total cresceram 18\% e $41 \%$, respectivamente, durante o mesmo período. Com base na análise, não há dúvida de que o consumo de frutose progrediu ao longo das últimas quatro décadas nos Estados Unidos, que os adolescentes e adultos jovens são os maiores consumidores, e que as bebidas adoçadas são as principais fontes alimentares de frutose ${ }^{[18]}$.

$\mathrm{Na}$ área urbana da África do Sul, foi estimado que $12,3 \%$ da energia da dieta sejam provenientes de açúcares de adição ${ }^{[19]}$. Nos Estados Unidos, Lewis et al. ${ }^{[8]}$, com base nos dados obtidos por meio da pesquisa 
National Health Knowledge Survey de 1977-1978, estimaram que $21 \%$ da energia provinham de açúcares naturais e adicionados, sendo que os açúcares de adição equivaliam a 56\% do total de açúcares ingerido e $11 \%$ do valor energético total. Considerando os dados da pesquisa Continuing Survey of Food Intake by Individuals (CFSII) realizada nos anos de 1989/1991 e 1994/1996, foi possível identificar que, respectivamente, 13,2 e 15,8\% da energia provinham de açúcares de adição ${ }^{[16]}$.

Thompson et al. ${ }^{[20]}$ relatam que na dieta de americanos com pelo menos dois anos, entre os anos de 1992 e 2002, a média alcançava 22,9 tsp/dia (cerca de $359 \mathrm{kcal}$, ou 16,6\% da energia de consumo diária) de açúcares de adição. Ainda segundo os autores, raça, renda familiar e grau de escolaridade estão independentemente associados com a ingestão de açúcares de adição. Foi observado que os grupos de baixa renda e educação são particularmente mais vulneráveis a dietas com expressiva adição de açúcares.

Comparativamente aos dados disponíveis para a população dos Estados Unidos, os demais países dispõem de menor acervo de informações, sendo a única fonte oficial disponível a base de dados elaborada pela Organização Internacional do Açúcar. Globalmente, a média mundial per capita de consumo do açúcar aumentou 16\%, quando se considera um período de 20 anos $(56 \mathrm{~g} /$ dia em 1986 para $65 \mathrm{~g} /$ dia em 2007). Em 1996, América do Sul e Oceania eram os maiores consumidores, seguidos pela Europa. Inversamente, o menor consumo era identificado na Ásia e continente africano. O consumo do açúcar aumentou em todas as partes do mundo, exceto Oceania, com crescimento mais impressionante na Ásia $(50 \%)^{[18]}$.

No Brasil, segundo dados estatísticos disponibilizados pelo Instituto Brasileiro de Geografia e Estatística (IBGE), utilizando-se como fonte a POF 1995-1996, estima-se um consumo médio de 4,34 $\mathrm{g} /$ dia de frutose livre, originária de frutas, doces, hortaliças e outros vegetais, e aproximadamente $27,5 \mathrm{~g} /$ dia provinda da sacarose ${ }^{[14]}$.

Avaliando-se os resultados de cinco pesquisas de âmbito nacional (ENDEF, realizada em 1974-1975, e POFs conduzidas em 1987-1988, 1995-1996, 20022003 e 2008-2009), foi observado que a disponibilidade de açúcar refinado ficou em torno de $15 \mathrm{~kg}$, em 1975 e 1988, com pequena queda em 1996 (quantidade anual per capita um pouco acima de $13 \mathrm{~kg}$ ). Já nas pesquisas seguintes, a quantidade média adquirida deste produto apresentou queda acentuada, passando para $8,3 \mathrm{~kg}$ na POF 2002-2003 e 3,3 kg na POF 2008-2009. Em comparação à aquisição média de 1975, a queda situase em $48 \%$ e $80 \%$, respectivamente. O açúcar cristal, cuja aquisição média em 2002-2003 era de 12,162 kg, apresentou quantidade média de 8,038 kg em 20082009 (redução de 33,9\%) ${ }^{[4]}$.

Entretanto, a evolução do consumo domiciliar de alimentos nos últimos seis anos (20032009) indica persistência de um teor excessivo de açúcar (com redução na participação do açúcar de mesa e incremento na fração oriunda de alimentos processados). A estabilidade da participação dos açúcares livres em torno de 17\% (muito acima do limite máximo de 10\%), em todos os estratos de rendimentos confirma a situação insatisfatória no consumo deste nutriente independentemente da renda familiar ${ }^{[4]}$.

Ao se avaliar o consumo de açúcares de adição entre os indivíduos mais jovens (crianças e adolescentes), inquérito (Health Behaviour in SchoolAged Children - HBSC) realizado bianualmente, em mais de 40 países, pela Organização Mundial da Saúde revelou que $35 \%$ dos adolescentes de 13 anos de idade consumiam frutas diariamente e $27 \%$ deles ingeriam algum tipo de refrigerante com igual frequência ${ }^{[21]}$.

Carmo et al. [22] avaliaram as práticas alimentares de adolescentes entre 10 e 17 anos de idade matriculados na rede pública de ensino da cidade de Piracicaba (São Paulo), por meio de questionário de frequência alimentar semiquantitativo. Os autores identificaram um consumo médio de 3,8 porções diárias de doces, sendo que $78,2 \%$ dos entrevistados ultrapassaram a recomendação máxima. O consumo médio diário alcançou aproximadamente $230 \mathrm{ml}$ e 550 $\mathrm{ml}$ de refrigerante e bebidas com adição de açúcar, respectivamente.

Por meio da Pesquisa Nacional de Saúde do Escolar foi identificada a frequência semanal de consumo de alimentos considerados como marcadores de alimentação saudável (feijão, legumes e verduras, frutas e leite) e de alimentação não saudável 
(frituras, embutidos, biscoitos e bolachas, guloseimas e refrigerantes). Observou-se que a proporção de escolares que consumiam guloseimas em cinco dias ou mais nos últimos sete dias que antecederam a coleta de dados foi de 50,9\% (total das capitais estudadas e Distrito Federal), variando de 41,8\%, em São Luís, a $56,8 \%$, em Goiânia. Já a frequência de escolares que ingeriram refrigerantes variou de 25,3\%, em São Luís, a 47,0\%, em Cuiabá. Notou-se ainda que o consumo de guloseimas superou o consumo de frutas frescas na totalidade das capitais e no Distrito Federal. Situação similar foi verificada (exceto em São Luís, Natal e Florianópolis) com o consumo de refrigerante ${ }^{[21]}$.

Um inquérito recordatório de 24 horas aplicado em uma sub-amostra de 104 indivíduos, de ambos os sexos, selecionados em estudo de base populacional e que considerou a frequência de citação e contribuição para o consumo de energia e nutrientes, identificou o refrigerante como um dos itens alimentares consumidos por mais de $50 \%$ da amostra e o grupo de açúcar/doces entre aqueles que mais contribuíram para a ingestão de carboidratos entre os homens ${ }^{[23]}$.

Cimadon et al. ${ }^{[24]}$ ao investigarem, em estudantes de Bento Gonçalves (RS), a prevalência de fatores de risco para aterosclerose com ênfase nos hábitos alimentares, mostraram que dentre os alimentos consumidos quatro ou mais vezes por semana, as guloseimas (doces, biscoitos recheados, chocolate e salgadinhos) e as bebidas açucaradas (refrigerantes comuns, sucos em pó, sucos concentrados e sucos naturais) estiveram presentes em $42,7 \%(n=252) \mathrm{e}$ $71,0 \%(n=419)$ das dietas, respectivamente.

Bezerra \& Sichieri [25] ao analisarem as características da alimentação fora do domicílio e os respectivos gastos, revelaram que a maior frequência dessa modalidade de consumo, no Brasil, recaía sobre o grupo dos refrigerantes, variando de $9 \%$ no Nordeste a $14 \%$ no Sudeste. Na região Sul, o item que apresentou maior frequência foi o das refeições e no Nordeste, o dos doces. Já o grupo com menor frequência de consumo fora do domicílio em todas as regiões foi o das frutas. Na POF 2008-2009, as despesas com aquisições de alimentos fora do domicílio apresentaram participação de 31,1\% no total das despesas com alimentação, significando um crescimento de $7,0 \%$ entre as duas últimas pesquisas
(POF 2002-2003 e 2008-2009) ${ }^{[4]}$.

\section{IMPACTO NA SAÚDE HUMANA}

O crescimento do consumo de frutose e a ampliação da incidência da obesidade na população americana levaram alguns pesquisadores a sugerirem que a dieta rica em frutose contribui decisivamente para o ganho de peso corporal. A frutose de fato possui características metabólicas que poderiam potencialmente contribuir para o aumento da adiposidade corporal e resistência à insulina (síndrome metabólica) ${ }^{[26]}$.

A presença de moléculas de glicose de pequeno tamanho (glicose, sacarose, lactose) estimula a síntese de ácidos graxos a nível hepático. Destaque se dá para a frutose, que estimula a $\alpha$ de novo lipogênese devido ao mecanismo direto de absorção pelo fígado que direciona este composto para o caminho da lipogênese hepática ${ }^{[27]}$. Desse modo, a ingestão de frutose induz um perfil endócrino que poderia favorecer o aumento da ingestão de energia e ganho de peso por atenuar os níveis de insulina e leptina, dois hormônios que inibem a ingestão de alimentos e contribuem para a regulação do balanço energético e adiposidade corporal, por meio de ações no sistema nervoso central. Além disso, o comprometimento da frutose-induzida na supressão pós-prandial da grelina, um hormônio que estimula a fome e a ingestão de alimentos, também podem contribuir para maior ingestão de alimentos ${ }^{[26]}$.

Pesquisas sobre o excesso de peso vêm sugerindo que o alto consumo de frutose $(>50 \mathrm{~g} / \mathrm{dia})$ poderia estar relacionado com o desenvolvimento da síndrome metabólica (obesidade, dislipidemia, hipertensão, resistência à insulina, estado pró-inflamatório, etc.) além de aumentar o risco de doenças cardiovasculares e possível desenvolvimento de demência, como por exemplo, doença de Alzheimer ${ }^{[28,29]}$.

Existem muitas evidências considerando que a ingestão elevada de frutose pode induzir a alterações do metabolismo, como o aumento de triglicérides e esteatose hepática. Esses efeitos são observados de forma evidenciada em ratos concomitantemente com o aumento da massa corporal. Em humanos, muitas dessas alterações são observadas em experimentos com quantidades de frutose que excedem largamente sua ingestão habitual e em condições hipercalóricas. 
Quando administrados como parte de uma dieta para manutenção do peso, a frutose pode de fato elevar os triglicérides plasmáticos. O consumo de frutose e bebidas com açúcar de adição está intimamente ligado e há uma forte evidencia de que elas favorecem o ganho e o excesso de peso e, por sua vez, ambas contribuam para o aumento dos riscos de diabetes e doenças coronarianas ${ }^{[30]}$.

Perez-Pozo et al. ${ }^{[31]}$ testaram a hipótese de que a frutose poderia induzir à síndrome metabólica, tendo como base amostral 74 homens adultos que consumiram $200 \mathrm{~g}$ de frutose por dia durante duas semanas. A ingestão de frutose resultou em aumento da pressão arterial, triglicérides, insulina de jejum e todos os testes da função hepática mostraram alterações nos valores. A proporção de indivíduos com síndrome metabólica aumentou (variação de 25$33 \%$ ). Os autores concluíram que a excessiva ingestão de frutose pode ser uma das causas da atual epidemia de obesidade e diabetes.

O aumento do uso do xarope de milho rico em frutose nos alimentos concomitantemente com o aumento da obesidade em americanos é mostrado na Figura 2.

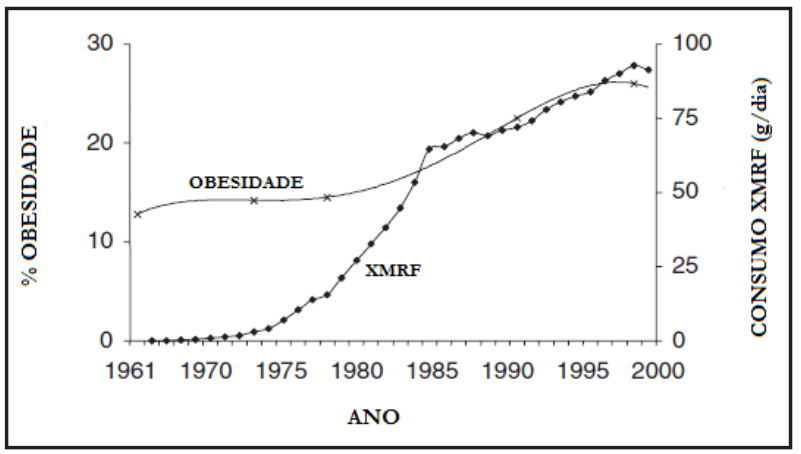

Figura 2. Ingestão do xarope de milho rico em frutose (XMRF) em função do aumento da prevalência da obesidade em americanos. Adaptado de Bray et al. ${ }^{[32]}$.

Teff et al. ${ }^{[26]}$ comparando os efeitos da frutose e da glicose consumidos (30\% do total de kcal) durante as refeições sobre os hormônios e substratos metabólicos de indivíduos obesos, mostraram que o consumo de frutose foi associado com baixa secreção de insulina e aumento de triglicérides pós-prandial nesses indivíduos quando comparado ao consumo de glicose. A elevação do triglicérides foi maior em indivíduos obesos com resistência insulínica, sugerindo que o consumo excessivo de frutose pode exacerbar um perfil metabólico adverso já presente em muitos indivíduos obesos.

Sartorelli et al. ${ }^{[33]}$ por meio de pesquisa que adotou o modelo transversal e amostra de 475 homens e 579 mulheres nipônico-brasileiros com idade de aproximadamente 30 anos, mostraram que elevadas ingestões de frutose e suco de frutas com açúcar de adição na dieta foram associados à intolerância à glicose entre indivíduos geneticamente susceptíveis. No entanto, não houve associação quando se considerou o elevado consumo de frutas frescas e a intolerância à glicose.

Estudo comparando os efeitos do consumo da frutose pura com adoçantes dietéticos, xarope de milho rico em frutose e sacarose, indicou que o xarope de milho rico em frutose e a sacarose em excesso aumentam o triglicérides pós-prandial em comparação com a frutose pura. Porém não sobram dúvidas que outros estudos que investiguem o consumo de frutose prolongado por si só ou em combinação com a glicose, sobre o metabolismo lipídico e resistência insulínica, na eutrofia, sobrepeso e obesidade são necessários ${ }^{[34]}$.

A ingestão de frutose em paralelo com o aumento da incidência de obesidade e diabetes são os principais fatores de risco para o desenvolvimento da Doença Hepática Gordurosa Não Alcoólica (DHGNA). Estudos com humanos sugerem que pacientes com DHGNA e particularmente aqueles com danos mais graves no fígado (esteatohepatite não alcoólica) consomem mais carboidratos e especialmente frutose em sua dieta em comparação com indivíduos saudáveis ${ }^{[35]}$.

Bomback et al. [36] realizaram pesquisa envolvendo períodos de 3 a 9 anos de acompanhamento de 15.745 pacientes e concluíram que o aumento do consumo regular de refrigerantes foi associado com hiperuricemia prevalente e doença renal crônica. A análise estratificada também sugeriu que a associação entre tais bebidas adoçadas e função renal alterada se deu principalmente entre os participantes com níveis elevados de ácido úrico. No entanto, em análises longitudinais, estas associações não foram identificadas. Os resultados apresentaram novos elementos de prova, mas divergiram se refrigerantes 
açucarados e, potencialmente, o xarope de milho rico em frutose usado para adoçá-los, representam um fator de risco dietético para o desenvolvimento de hiperuricemia e doença renal crônica.

Douard et al. ${ }^{[37]}$ por meio de estudo experimental, envolvendo ratos, já com a função renal comprometida, alimentados com uma dieta com $60 \%$ de frutose durante seis semanas, mostraram que a ingestão de frutose dietética em excesso inibe a absorção intestinal de cálcio e induz a insuficiência de vitamina $\mathrm{D}$ na doença renal crônica, além de agravar outros sintomas clássicos da mesma.

A relação entre o consumo de refrigerantes e fatores cardiovasculares avaliada em 6.039 participantes de uma pesquisa (Framingham Heart Study) revelou que o consumo de mais de uma lata de refrigerante $(350 \mathrm{ml})$ por dia foi significativamente associada à prevalência da síndrome metabólica. Além disso, nos indivíduos sem a referida morbidade no momento da inclusão na pesquisa, o consumo de mais de um refrigerante por dia foi associado com um aumento no risco para o desenvolvimento da síndrome metabólica ${ }^{[38]}$.

Tendo por base pesquisa envolvendo 88.520 mulheres, a ingestão de bebida com açúcar de adição foi significativamente associada com doenças do coração ${ }^{[39]}$. Foi observada também que a relação entre a ingestão de bebidas com açúcar de adição e incidência de doença arterial coronariana permaneceu significativa, mesmo após o ajuste para peso corporal e, pode ser atribuída tanto ao maior índice glicêmico como ao alto teor de frutose das bebidas ingeridas.

Os refrigerantes integram a cultura de diversos países, e seu consumo tem aumentado constantemente nos últimos 50 anos. Refrigerantes com aproximadamente 560 gramas de xarope de milho rico em frutose tem cerca de $250 \mathrm{kcal}$. Assim, um refrigerante com uma quantidade extra de 560 gramas por dia é mais do que suficiente para dar conta do aumento do peso corporal durante o último quarto de século. Atualmente, os refrigerantes são parte importante da cultura do fast food. Quando as pessoas optam pelo consumo em um restaurante do tipo fast food, em comparação ao dia em que não o fazem, consomem uma maior quantidade de refrigerantes e batatas fritas e, menor consumo de cereais, legumes, leite e derivados ${ }^{[40]}$.

Com base nos estudos disponíveis, parece haver evidências, em modelos animais, de que o consumo de frutose pode induzir a resistência à insulina, tolerância à glicose diminuída, hiperinsulinemia, hipertrigliceridemia e hipertensão ${ }^{[41]}$. Porém, não existem, até o momento, evidências de efeitos semelhantes em seres humanos em padrões de consumo médios $(50 \mathrm{~g} / \mathrm{dia})$. Apesar de existirem análises mostrando os efeitos metabólicos e endócrinos do crescimento do consumo de frutose e o aumento ponderal, adiposidade corporal e resistência à insulina, é necessário maior número de investigações para esclarecer completamente o efeito metabólico da frutose em seres humanos. Em expressiva parcela dos estudos epidemiológicos, transversais ou longitudinais, a existência de correlação positiva entre o consumo de refrigerantes e o excesso de peso ainda não pôde ser confirmada.

Respostas metabólicas à frutose podem ser afetadas por hormônios (como corticóides e catecolaminas) liberados em resposta ao estresse, e os resultados de estudos a curto prazo realizados com grandes doses de frutose ( $>100 \mathrm{~g} /$ dia) parecem não prever com precisão respostas que podem ocorrer com a ingestão da mesma quantidade de frutose distribuídas ao longo do dia ${ }^{[42]}$.

Embora venha ocorrendo o acentuado aumento da obesidade e consumo de frutose na população, não há evidências diretas relacionando a obesidade à ingestão de quantidades fisiológicas de frutose em seres humanos $(\leq 100 \mathrm{~g} /$ dia $)$. O consumo de uma dose moderada de frutose $(\leq 50 \mathrm{~g} / \mathrm{dia}$ ou aproximadamente $10 \%$ do total de calorias da dieta) parece não provocar qualquer efeito deletério na concentração de triglicérides de jejum e pós-prandial, controle glicêmico e resistência à insulina. Também parece não influenciar na ingestão de alimentos e no peso corporal e não há nenhuma evidência da relação entre o consumo moderado de frutose e hipertensão. Portanto, não é possível afirmar, com base na literatura disponível, a relação direta entre a ingestão moderada de frutose na dieta e marcadores de risco para a saúde ${ }^{[41,42]}$.

\section{RECOMENDAÇÃO DIETÉTICA}

\section{Em 2003, a World Health Organization} preconizou que a ingestão de açúcar não superasse 10\% do total energético diário. Em 2005, a American Dietary Guidelines recomendou a diminuição do 
consumo de alimentos ricos em açúcares de adição, limitando o total de energia para proporções entre $8 \%$ e $20 \%$, dependendo do total energético gasto por indivíduo. O Institute of Medicine, por meio das Dietary Reference Intakes, recomendou que a maior parte da energia deve ser obtida a partir do total dos grupos de alimentos e menor conteúdo energético deve estar disponível para o uso em forma de açúcares de adição ${ }^{[20]}$.

A American Heart Association publicou as diretrizes para a ingestão de açúcar de adição na dieta, recomendando que o consumo de açúcar seja limitado a 100 e $150 \mathrm{kcal} /$ dia para homens e mulheres, respectivamente ${ }^{[43]}$.

O guia alimentar da Associação Americana de Cardiologia (AHA) lançado em 2006 recomenda o consumo semanal de no máximo cinco porções de alimentos fontes de açúcares de adição ${ }^{[4]}$.

A Organização Mundial da Saúde preconiza que o consumo de açúcares livres não ultrapasse a 10\% do valor energético total (VET) da dieta, proporção justificada pelas evidências de que a prevalência de cáries dentais é baixa em países onde o consumo de açúcares não ultrapassa $20 \mathrm{~kg}$ per capita por ano. Isto equivale a 40-55 gramas per capita/dia ou 6-10\% do VET [2].

No guia alimentar brasileiro elaborado pelo Ministério da Saúde ${ }^{[10]}$, a recomendação do consumo de açúcares é de 10\% do valor energético total da dieta. Neste documento é estipulado o consumo diário máximo de uma porção de doces e açúcares.

Vale ressaltar, que até o presente momento, não foi disponibilizada nenhuma recomendação de ingestão específica para a frutose, e que o consumo da mesma proveniente de frutas e hortaliças não deve ser desencorajado, pois sua ingestão é considerada segura e saudável.

\section{CONSIDERAÇÕES FINAIS}

Estudos vêm reconhecendo que uma alimentação rica em frutose pode elevar o triglicérides plasmático e o colesterol total. Dados recentes sugerem também que o excessivo consumo de frutose por seres humanos pode resultar em aumento da adiposidade visceral, desregulação de lipídeos e diminuição da sensibilidade à insulina, aumentando o risco para doenças cardiovasculares, diabetes tipo 2 e obesidade. Porém, as implicações dos eventuais efeitos da frutose sobre diferentes aspectos do metabolismo em termos de possíveis riscos para a saúde humana devem ser determinados por meio de estudos de intervenção a longo prazo, delineados de forma rigorosa, e que considerem os efeitos à saúde do consumo de frutose em quantidades habitualmente ingeridas pelos indivíduos

A formulação de legislação e implementação de políticas públicas para limitar o consumo dos alimentos ricos em açúcares de adição tais como aquelas modalidades envolvendo ações de educação nutricional e o acesso as informações sobre a quantidade de açúcares nos rótulos dos alimentos, poderiam fornecer à população subsídios para a realização de escolhas alimentares mais saudáveis, o que obviamente ampliaria também o acesso à segurança alimentar e nutricional.

\section{REFERÊNCIAS}

1. Food and Agriculture Organization/World Health Organization. Fruit and vegetables for health: Report of a Joint FAO/WHO Workshop, 1-3 September, 2004, Kobe, Japan. Geneva; 2005. 39 p.

2. World Health Organization. Diet, nutrition and the prevention of chronic diseases. Geneva, 2003. 160 p. (WHO. Technical Report Series, 916).

3. Murphy SP, Johnson RK. The scientific basis of recent US guidance on sugars intake. Am J Clin Nutr. 2003;78(4):82733.

4. Instituto Brasileiro de Geografia e Estatística. Pesquisa de Orçamentos Familiares 2008-2009: aquisição alimentar domiciliar per capita - Brasil e grandes regiões. Rio de Janeiro: IBGE; 2010.

5. Levy-Costa RB, Sichieri R, Pontes NS, Monteiro CA. Disponibilidade domiciliar de alimentos no Brasil: distribuição e evolução (1974-2003). Rev Saúde Públ. 2005;39(4):530-40.

6. Rinaldi ERA, Pereira AF, Macedo CS, Mota JF, Burini RC. Contribuições das práticas alimentares e inatividade física para o excesso de peso infantil. Rev Paul Pediatr. 2008;26(3):271-77. 
7. Brand-Miller JC, Foster-Powell E, Colagiuri F. A nova revolução da glicose. Rio de Janeiro: Editora Campus; 2003.

8. Lewis CJ, Park YK, Dexter PD, Yetley EA. Nutrient intake and body weights of persons consuming high and moderate level of added sugars. J Am DietAssoc. 1992;92(6):708-13.

9. Boowman S. Diet of individuals based on energy intakes from added sugars. US Department of Agriculture, Center for Nutrition Policy and Promotion. Fam Econ Nutr Rev. 1999;12:31-8.

10. Brasil. Ministério da Saúde. Secretaria de Atenção à Saúde. Coordenação-Geral da Política de Alimentação e Nutrição. Guia alimentar para a população brasileira: promovendo a alimentação saudável. Brasília: Ministério da Saúde; 2006. 210 p. (Série A. Normas e Manuais Técnicos).

11. United States Department of Agriculture and U. S. Department of Health and Human Service. Dietary Guidelines for Americas; 2005. 6th Edition. 84 p.

12. Angelis K, Schaan BA, Rodrigues B, Malfitano C, Irigoyen MC. Disfunção autonômica cardiovascular no diabetes mellitus experimental. Arq Bras Endocrinol Metab. 2007;51(2):185-94.

13. Dutra-de-Oliveira JE, Marchini JS. Ciências Nutricionais. São Paulo: Sarvier; 2008.

14. Barreiros RC, Bossolan G, Trindade CEP. Frutose em humanos: efeitos metabólicos, utilização clínica e erros inatos associados. Rev Nutr. 2005;18(3):377-89.

15. Ferreira VF, Rocha DR. Potencialidades e oportunidades na química da sacarose e outros açúcares. Quím Nova. 2009;32(3):623-38.

16. Guthrie J, Morton JF. Food sources of added sweeteners in the diets of Americans. J Am Diet Assoc. 2000;100(1):43-5.

17. Institute of Medicine. Dietary reference intakes for energy, carbohydrates, fiber, fat, protein and amino acids (macronutrients).Washington: National Academy Press, 2002.

18. Tappy L, Le K. Metabolic effects of fructose and the worldwide increase in obesity. Physiol Rev. 2010;90(1):23-46.

19. Steyn NP, Myburgh NG, Nel JH. Evidence to support a food-based dietary guideline on sugar consumption in South Africa. Bull Word Health Organ. 2003;81(8):599-608.

20. Thompson FE, Mcneel TS, Dowling EC, Midthune D, Morrissette M, Zeruto CA. Interrelationships of added sugars intake, socioeconomic status, and race/ethnicity in adults in the United States: National Health Interview Survey, 2005. J Am Diet Assoc. 2009;109(8):1376-83.

21. Instituto Brasileiro de Geografia e Estatística. Pesquisa Nacional de Saúde do Escolar. Rio de Janeiro, 2009 [acesso em 11 nov 2011]. Disponível em: http://portal. saude.gov.br/portal/arquivos/pdf/livro pense.pdf

22. Carmo MB, Toral N, Silva MV, Slater B. Consumo de doces, refrigerantes e bebidas com adição de açúcar entre adolescentes da rede pública de ensino de Piracicaba, São Paulo. Rev Bras Epidemiol. 2006;9(1):121-30.

23. Ferreira MG, Silva NF, Schmidt FD, Silva RMVG, Sichieri R, Guimarães LV et al. Desenvolvimento de questionário de frequência alimentar para adultos em amostra de base populacional de Cuiabá, Região Centro-Oeste do Brasil. Rev Bras Epidemiol. 2010;13(3):413-24.

24. Cimadon HMS, Geremia R, Pellanda LC. Hábitos alimentares e fatores de risco para aterosclerose em estudantes de Bento Gonçalves (RS). Arq Bras Cardiol. 2010;95(2):166-72.

25. Bezerra IN, Sichieri R. Características e gastos com alimentação fora do domicílio no Brasil. Rev Saúde Públ. 2010;44(2):221-29.

26. Teff KL, Grudziak J, Townsend RR, Dunn TN, Grant RW, Adams SH et al. Endocrine and metabolic effects of consuming fructose and glucose-Sweetened beverages with meals in obese men and women: influence of insulin resistance on plasma triglyceride responses. J Clin Endocrinol Metab. 2009;94(5):1562-69.

27. Silvestre R. O argumento para a inclusão de uma dieta com baixos valores de hidratos de carbono no tratamento e prevenção da síndrome metabólica. Revista Portuguesa de Endocrinologia, Diabetes e Metabolismo. 2008;3(1):59-72.

28. Stephan BCM, Wells JCK, Brayne C, Albanese E, Siervo M. Increased fructose intake as a risk factor for dementia. J Gerontol A Biol Sci Med Sci. 2010;65(8):809-14. 
29. Johnson RJ, Murray R. Fructose, exercise and health. Curr Sports Med Rep. 2010;9(4):253-58.

30. Tappy L, Le KA, Tran C, Paquot N. Fructose and metabolic diseases: new findings, new questions. Nutrition. 2010; 26(11):1044-49.

31. Perez-Pozo SE, Schold J, Nakagawa T, Sanchez-Lozada LG, Johnson RJ, Lillo L. Excessive fructose intake induces the features of metabolic syndrome in healthy adult men: role of uric acid in the hypertensive response. Int $\mathrm{J}$ Obes. 2010; 34(3):454-61.

32. Bray GA, Nielsen SJ, Popkin BM. Consumption of highfructose corn syrup in beverages may play a role in the epidemic of obesity. Am J Clin Nutr. 2004;79(4):537-43.

33. Sartorelli DS, Franco LJ, Gimeno SGA, Ferreira SRG, Cardoso MA. Dietary fructose, fruits, fruit juices and glucose tolerance status in Japanesee-Brazilians. Nutr Metab Cardiovasc Dis. 2009;19(2):77-83.

34. Stanhope KL, Havel PJ. Fructose consumption: recent results and their potential implications. Ann N Y Acad Sci. 2010; 1190:15-24.

35. Spruss A, Bergheim I. Dietary fructose and intestinal barrier: potential risk factor in the pathogenesis of nonalcoholic fatty liver disease. J Nutr Biochem. 2009;20(9):657-62.

36. Bomback AS, Derebail VK, Shoham DA, Anderson CA, Steffen LM, Rosamond WD. Sugar-sweetened soda consumption, hyperuricemia, and kidney disease. Kidney Int. 2010;77(7):609-16.

37. Douard V, Asgerally A, Sabbagh Y, Sugiura S, Shapses SA, Casirola D et al. Dietary fructose inhibits intestinal calcium absorption and induces vitamin D insufficiency in CKD. J Am Soc Nephrol. 2010;21(2):261-71.

38. Dhingra R, Sullivan L, Jacques PF, Wang TJ, Fox CS, Meigs JB et al. Soft drink consumption and risk of developing cardiometabolic risk factors and the metabolic syndrome in middle-aged adults in the community. Circulation. 2007;116(5):480-88.

39. Fung TT, Malik V, Rexrode KM, Manson JE, Willett WC, Hu FB. Sweetened beverage consumption and risk of coronary heart disease in women. Am J Clin Nutr. 2009;89(4):1037-42.
40. Bray GA. Fructose: should we worry? Int J Obes. 2008;32(Suppl. 7):127-31.

41. Rizkalla SW. Health implications of fructose consumption: a review of recent data. Nutr Metab. 2010; 7(82):1-17.

42. Dolan LC, Potter SM, Burdock GA. Evidence-based review on the effect of normal dietary consumption of fructose on blood lipids and body weight of overweight and obese individuals. Crit Rev Food Sci Nutr. 2010;50(10):889-918.

43. Johnson RK, Appel LJ, Brands M, Howard BV, Lefevre M, Lustig RH et al. Dietary sugars intake and cardiovascular health: a scientific statement from the American Heart Association. Circulation. 2009;120(11):1011-20.

44. Lichtenstein AH, Appel LJ, Brands M, Carnethon M, Daniels S, Franch HA et al. Diet and lifestyle recommendations revision 2006: a scientific statement from the American Heart Association Nutrition Committee. Circulation. 2006;114(1):82-96. 